\title{
A comparison of rapid methods including imprint cytodiagnosis for the diagnosis of breast tumours
}

\author{
C. R. TRIBE 1
}

From the Department of Pathology, Wycombe General Hospital, High Wycombe, Bucks

SYNOPSIS Rapid diagnoses were made on 510 breast lumps by three methods in sequence and the results compared with those obtained by paraffin histology.

Naked-eye examination of the excised lumps correctly distinguished between benign and malignant tumours in $95.1 \%$ of cases with $23(4.5 \%)$ false negative and two $(0.4 \%)$ false positive results.

Rapid imprint cytodiagnosis was correct in $96.9 \%$ of the 391 lumps imprinted with $12(3 \cdot 1 \%)$ false negatives and no false positive results.

Frozen sections gave the correct diagnosis in $99.4 \%$ of the 510 cases with three $(0.6 \%)$ false negative and no false positive results.

The use of cytology in the diagnosis of breast tumours is discussed.

Examination of frozen sections has been the standard method of rapid diagnosis of breast tumours for many years and with the advent of the refrigerated microtome is now virtually as accurate as study of paraffin sections. However, facilities for frozen sections are not always available and in these circumstances a surgeon may proceed to mastectomy relying on the clinical findings and the characteristic naked-eye features of carcinoma found in the breast biopsy. Although little used in this country, rapid cytological diagnostic methods are available either by examining smears prepared from needle aspirates of breast lumps (Gibson and Smith, 1957; Webb, 1970) or smears and imprints made from the excised lumps (Dudgeon and Barrett, 1934; Tribe, 1965).

In a previous paper (Tribe, 1965) cytological diagnosis of breast tumours by the imprint method was evaluated by a retrospective study of permanently stained imprints made from 311 breast tumours and a high degree of accuracy was obtained. At that time a rapid impermanent staining method using polychromatic methylene blue was described which took only two minutes. Using this method a cytological diagnosis can be made before examining the frozen section. In this study a 'comparison was made between the diagnoses of breast tumours obtained by four different methods: (1) naked-eye

'Present address: Department of Pathology, Southmead Hospital, Westbury-on-Trym, Bristol.

Received for publication 26 January 1973. diagnosis made by the pathologist from examination of the excised breast lump; (2) cytological diagnosis from imprints stained by the rapid method; (3) microscopic examination of frozen sections; and (4) microscopic examination of paraffin sections.

These four methods were used consecutively on each breast lump studied and a definite diagnosis of benign or malignant was recorded at each stage. An analysis and comparison of the results obtained from each method will be presented and the advantages and disadvantages of using these methods alone or in combination will be discussed.

It should be appreciated that with one pathologist alone involved in this study, only the first of these methods, the naked-eye diagnosis from examination of the excised lump, was carried out blind and independently of the other methods. In the other three methods employed knowledge of the previous examinations will inevitably have led to some observer bias when recording the subsequent results. Therefore comparison of the methods as presented is not as valid as it would have been if the smears and sections had been read independently of each other and of the macroscopic findings. The previous study (Tribe, 1965) had shown that a high degree of diagnostic accuracy could be obtained solely by use of the imprint method. This study was deliberately intended to test the use of rapidly stained imprints when introduced into routine urgent examination of frozen sections of breast lumps by one pathologist. It also tested the accuracy of naked-eye and 
cytological examinations without the use of histological methods.

\section{Material and Methods}

In the district served by this department between 1966 and 1970 rapid histological diagnosis by means of frozen section at the time of operation was made on 510 breast tumours ('lumps in the breast'). The final diagnoses from paraffin sections showed that 226 of these tumours were malignant and 284 were benign.

In each case the pathologist made a naked-eye diagnosis from examination of the excised lump before examining imprints or the frozen sections. For the purpose of this paper these macroscopic diagnoses have been limited to 'malignant' or 'benign' and in any case in which the naked-eye appearance was not diagnostic it was recorded as benign. This distinction was made in order to make the results equivalent to the decision taken by a surgeon whether or not to proceed to mastectomy purely from naked-eye examination of the breast biopsy.

\section{METHOD RECOMMENDED FOR EXAMINING EXCISED BREAST LUMPS}

Palpation, section, re-palpation, and inspection in that order are suggested as the best routine for naked-eye examination of the freshly removed breast lump. Palpation is the most important examination since carcinoma of the breast is almost without exception uniquely hard. The only lesion to feel as hard as cancer is a thick-walled cyst and this becomes obvious after section when the hardness disappears. Inspection after cutting the lump will often help in diagnosing the type of tumour concerned. The lobulated, rather friable, whorled appearance of the fibroadenoma is unmistakable, and the retracted, gritty, 'raw pear' appearance of the scirrhous carcinoma is also usually diagnostic. The better delineated, greyer, protruding medullary carcinoma can usually be distinguished from other malignant tumours (Richardson, 1956).

In $391(76.7 \%)$ cases an imprint stained by the rapid method was examined and a diagnosis of malignant or benign made before the frozen section was studied. Imprints were not prepared in all cases since a proportion of the benign lumps were $s 0_{*}^{*}$ obviously innocuous that cytological study did not appear necessary. This accounts for the higher $\overrightarrow{\vec{D}}$ proportion of imprints made from malignant tumours, viz, 200 out of 226 malignant lumps imprinted $(88.5 \%)$ as compared with 191 out of $284 \overline{\bar{\rho}}$. benign lumps $(67 \cdot 3 \%)$.

In 126 out of the 200 imprints made from malignant lumps an attempt was made to grade the ${ }^{\text {s }}$ tumours and these results were compared with the $\overrightarrow{0}$ subsequent histological grades made according to the criteria laid down by Patey and Scarff (1928 and 1929), Bloom (1950), and Bloom and Richardson (1957).

Half the 510 frozen sections were prepared on a Pearse-Slee refrigerated microtome (cryostat) and ${ }^{+}$ the other half were cut on a standard frozen section apparatus adapted for use in small hospitalso (Casling and Tribe, 1969).

The methods for making and staining imprints $\vec{\square}$ from breast tumours and the cytological criteriao used for diagnosis were similar to those previously described (Tribe, 1965). Since this study made special use of the rapid method for staining imprints. ${ }^{\omega}$ it is worth repeating the staining details:

1 Flood the air-dried imprint with $1 \%$ polychromatic methylene blue for 30 to 60 seconds, depending on the rapidity of the particular batch of stain.

2 Wash with tap water.

3 Mount wet with a coverslip, squashing out the excess air bubbles.

\section{Results}

The overall results are summarized in table $\mathbf{I}$.

Although it was anticipated that each additionalo diagnostic method would increase the overall? accuracy in each case, it was surprising how oftenô the simpler methods of diagnosis were correct. As shown in table I the diagnosis from naked-eye examination of the 510 breast lumps was correct in $>$ 19 out of every 20 cases and of the $25(4.9 \%)$ false results only two $(0.4 \%)$ were false positives. Of then 391 lumps in which rapid imprint cytology was used, $379(96.9 \%)$ were correctly diagnosed and there $N$ were only $12(3.1 \%)$ false negatives with no false $\omega$ positives. Examination of the 510 frozen sections

\begin{tabular}{|c|c|c|c|c|c|}
\hline \multicolumn{2}{|c|}{ Type of Examination } & \multirow{2}{*}{$\begin{array}{l}\text { No. of Lumps } \\
510 \\
391 \\
510 \\
510\end{array}$} & $\begin{array}{l}\text { No. Correctly } \\
\text { Diagnosed }\end{array}$ & \multicolumn{2}{|c|}{ False Negatives False Negatives } \\
\hline $\begin{array}{l}\text { A } \\
\text { B } \\
\text { C } \\
\text { D }\end{array}$ & $\begin{array}{l}\text { Naked-eye assessment of breast lump } \\
\text { Rapid cytology } \\
\text { Frozen sections } \\
\text { Paraffin sections }\end{array}$ & & $\begin{array}{ll}485 & (95.1 \%) \\
379 & (96.9 \%) \\
507 & (99.4 \%)\end{array}$ & $\begin{array}{cc}23 & (4.5 \%) \\
12 & (3.1 \%) \\
3 & (0.6 \%) \\
\text { Assumed to show }\end{array}$ & $\begin{array}{ll}2 & (0 \cdot 4 \%) \\
0 & 0 \\
0 & 0 \\
\text { diagnosis }\end{array}$ \\
\hline \multicolumn{6}{|c|}{ Table I Overall results } \\
\hline
\end{tabular}


further increased the accuracy of diagnosis with only three $(0.6 \%)$ false negatives and no false positives.

Each method will now be evaluated with analysis of the false results.

NAKED-EYE ASSESSMENT OF BREAST LUMPS

This method proved to be surprisingly accurate. A busy general surgeon will soon gain enough experience to be quite confident of diagnosing the 'typical' malignant breast lump. A pathologist, depending on his work load, will probably gain experience of the naked-eye appearances of breast lumps at three to four times the rate of any one surgeon. In this series, apart from fibroadenomata, it was much easier to be certain that a breast lump was malignant than to be sure it was benign. The maxim 'difficult naked-eye, difficult on microscopy' was often proven correct.

The most difficult breast lump to diagnose macroscopically is undoubtedly the diffuse fibrotic microcystic mass from which small worms of putty-like material can often be expressed. This lesion is also often difficult to diagnose on both frozen and paraffin sections and includes the group of atypical hyperplasias thought by some authors to be premalignant in nature (Stein, 1967; Black and Chabon, 1969).

As shown in table I false negative diagnoses were made on naked-eye examination of 23 out of the 510 breast lumps in this series $(4.5 \%)$. These were either considered benign or not sufficiently abnormal to be certain of carcinoma. On further analysis of these cases 13 were recorded as doubtful or not diagnostic and only 10 were definitely recorded as benign. Two of the latter proved to be medullary carcinomata and one a mucoid carcinoma; both these tumour types have atypical macroscopic appearances.

There were only two false positive diagnoses. One was a lump removed from a 56-year-old woman in which, although no malignant cells were seen on imprint cytology and no definite invasive cancer demonstrated on frozen section, there was sufficient atypical intraduct hyperplasia in the paraffin sections to justify local mastectomy at a later date. The other false positive result occurred in a 34-year-old woman. Frozen section showed a benign intraduct papilloma and paraffin sections revealed additional benign florid sclerosing adenosis.

\section{RAPID IMPRINT CYTOLOGY}

As explained in the previous paper (Tribe, 1965), it is much easier to diagnose a breast tumour as malignant on imprint cytology than to be certain it is benign. It should also be remembered that there was no time to examine a second imprint if the first was unsatisfactory, since examination and diagnosis from the imprint was made before examining the frozen section. In spite of these limitations the results were more accurate than those obtained in the previous retrospective study. This was partially due to the advantage of having studied the naked-eye appearances of the breast lump immediately before looking at the imprint and also due to greater experience in making the imprints. Special attention was taken in trimming and drying the surface of the breast lump to be imprinted to avoid fat and excess tissue fluid which were the cause of a large proportion of the false negatives in the previous study.

For reasons previously explained, rapid imprint cytology was performed on only 391 out of the 510 breast lumps. Amongst these there were only 12 $(3.1 \%)$ false negative results and no false positives. On analysis, nine out of the 12 were considered to be poor imprints and not diagnostic and only three were definitely thought to be benign.

Imprints were examined on 19 out of the 25 lumps with false results in the naked-eye group. Seven gave similar false results to the naked-eye diagnosis but in 12 cytology established the correct diagnosis. It is considered that with a combination of naked-eye examination and imprint cytology a high degree of diagnostic accuracy can be obtained and the number of false results obtained from macroscopic study alone could be halved.

Ten cases in this series were reported as premalignant atypical cystic hyperplasia (carcinoma in situ) after examining many paraffin sections. It is worth noting that eight out of the nine imprints made from these 10 cases were considered to be benign.

In 126 out of the 200 imprints made from malignant lumps the tumours were graded as having a low, intermediate, or high degree of malignancy, according to the cytological criteria described in the previous paper. The results were compared with grades made from the equivalent paraffin sections according to the criteria established by Bloom (1950). The latter were performed without prior knowledge of the cytological grades. The results are expressed in table II which shows the concordant results in the oblique line from the top left to the bottom right.

Study of this table shows that although there was

\begin{tabular}{lccccc}
\hline \multicolumn{7}{l}{ From Paraffin Sections } \\
\cline { 2 - 6 } & Grade $^{1}$ & \multicolumn{1}{l}{ I } & II & III & Totals \\
\hline From & I & 29 & 12 & 1 & 42 \\
Imprints & II & 11 & 22 & 7 & 40 \\
& III & 1 & 8 & 35 & 44 \\
& Totals & 41 & 42 & 43 & 126 \\
\hline
\end{tabular}

Table II Grading of 126 breast carcinomata

${ }^{1}$ Grades of malignancy: I = low; II = intermediate; III = high 
a very close correlation in the total number of tumours placed in each grade by the two methods, only 86 out of the $126(68 \%)$ shows concordant results. In 38 tumours the grades varied by one degree and in two cases by two degrees. Both sets of results showed a slight increase in the number of tumours placed in the low or high grades of malignancy in comparison with Bloom's results, and this reflects the author's inclination to avoid the natural tendency to grade tumours into the 'safe' intermediate group. This small series shows that grading of tumours by cytological criteria broadly agrees with the equivalent histological grading and it is thought that further, longer study of permanently stained imprints could improve the correlation of the grading methods.

\section{FROZEN SECTIONS}

There were only three false negatives and no false positive results in the 510 frozen sections. These results compare favourably with the published results of larger frozen section series briefly reviewed in the previous paper and show an improvement in comparison with the five false negative and no false positive results obtained in 310 breast lumps in 1965. This reflects the greater use of cryostat sections in the present series.

The three false results in the present series were made on lumps which were all considered to be benign on naked-eye examination. Two had nondiagnostic imprints and all three tumours proved to be low-grade scirrhous carcinomata on paraffin sections.

\section{PARAFFIN SECTIONS}

The diagnoses made from the paraffin sections were assumed to be correct. The definitive diagnoses made from the paraffin sections on the 510 breast lumps in this series were as in table III.

\begin{tabular}{|c|c|c|c|c|c|c|c|c|c|}
\hline \multirow{2}{*}{\multicolumn{4}{|c|}{$\begin{array}{l}\text { Carcinoma (all types) } \\
\text { Cystic hyperplasia (all types) }\end{array}$}} & $\cdots$ & $\cdots$ & $\cdots$ & $\cdots$ & & 226 \\
\hline & & & & . & . & $\cdots$ & $\cdots$ & . & 210 \\
\hline Fibroade & ma & .. & $\ldots$ & . & . & . & $\ldots$ & .. & 50 \\
\hline Gynaeco & istia & $\ldots$ & . & . & . & . & $\ldots$ & .. & 12 \\
\hline Abscess & . & . & . & . & . & . & $\ldots$ & . & 9 \\
\hline Lipoma & .. & .. & . & . & . & $\cdots$ & . & $\cdots$ & $\begin{array}{r}3 \\
510\end{array}$ \\
\hline
\end{tabular}

Table III Diagnosis by paraffin sections

${ }^{1}$ Includes one case of giant fibroadenoma judged to be malignant on imprint cytology and frozen section. Local mastectomy was required to remove the large tumour.

'Includes 10 lumps judged as premalignant hyperplasia without invasion.

\section{Discussion}

It is worth stressing that in 19 out of every 20 breast

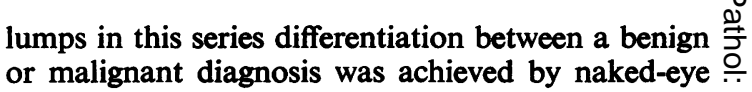
examination alone. It is suggested that in regions $\overrightarrow{\vec{\rho}}$ where frozen section facilities are not available a $\stackrel{\rho}{?}$ surgeon can soon learn to place great reliance on his naked-eye examination of the excised breast lump. If he is prepared to learn the relatively simple technique of cytodiagnosis from breast imprints with the rapid staining method he will be unlikely to perform a mastectomy on a benign lesion.

Using the cytological criteria suggested by the author, Webb (1970) described the use and indica- $\vec{\omega}$ tions for fine needle aspiration of breast lumps in surgical outpatients. He obtained $97 \cdot 3 \%$ accuracy ? of cytology in 108 out of 168 patients in whom $N$ histological confirmation was available. This author $\stackrel{\oplus}{\oplus}$ confirmed the high degree of accuracy which can be $N$ obtained from cytodiagnosis in breast cancer and it seems reasonable to proceed direct to mastectomy $\stackrel{\circ}{\supset}$ without frozen section on a patient with a cytological diagnosis of malignancy made from a fine needle aspirate.

Benign breast lesions, however, present a different $\vec{\theta}$ problem. There is ample evidence to show that $\omega$ fibrocystic disease of the breast with epithelial hyperplasia is common in patients with breast cancer and many authors believe in a more direct relationship. Although not all histopathologists are prepared to diagnose carcinoma in situ in the breast, Stein (1967) and Black and Chabon (1969) have recently classified the histological criteria of this diagnosis and its relationship to fibrocystic disease. One way in which histopathologists can help to reduce the overwhelming problem of mammary carcinoma is to try and select patients with fibrocystic disease in whom there is a strong possibility that carcinoma may develop. Ten patients in this series had sufficiently atypical changes in their breast biopsies to be classified as premalignant $₹$ hyperplasia and after full discussion with the 을 surgeons concerned several had local prophylactic $\rightarrow$ mastectomies although there was no histological o․ evidence of invasive carcinoma. As noted before, $\bar{N}$ imprint cytology failed to detect these lesions which $\%$ required prolonged study of many paraffin sections $N$ in each case. It seems that, unlike carcinoma in situ of the cervix, cytology appears to have no place in $\bar{\sigma}$ diagnosing premalignant changes in the breast.

This study has shown that benign breast lesions $\frac{0}{\infty}$ are very unlikely to be diagnosed as malignant after $\stackrel{\mathcal{C}}{\rightarrow}$ a combination of naked-eye examination and 0 imprint cytology from the excised breast lump.

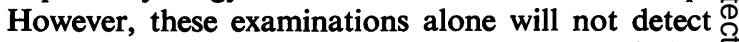
premalignant changes and before any breast lesion $\underset{\mathbb{D}}{\mathbb{Q}}$ is declared benign a full paraffin study of the excised breast lump should be undertaken. 
I wish to thank all the general surgeons in the High Wycombe and Amersham areas for their assistance and tolerance in this study, Mr Martin Casling and his colleagues for their technical aid, and Miss Jill Smallwood for her secretarial assistance.

\section{References}

Black, M. M., and Chabon, Ada B. (1969). In situ carcinoma of the breast. In Pathology Annual, pp. 185-210. Butterworths, London.

Bloom, H. J. G. (1950). Prognosis in carcinoma of the breast. Brit. J. Cancer, 4, 259-288.

Bloom, H. J. G., and Richardson, W. W. (1957). Histological grading and prognosis in breast cancer. Brit. J. Cancer, 11, 359-377.

Casling, M. F., and Tribe, C. R. (1969). Use of dichloro-difluoro methane aerosols in preparing frozen sections. J. clin. Path., 22, 244-245.

Dudgeon, L. S., and Barrett, N. R. (1934). The examination of fresh tissue by the wet-film method. Brit. J. Surg., 22, 4-22.

Gibson, A., and Smith, G. (1957). Aspiration biopsy of breast tumours. Brit. J. Surg., 45, 236-239.

Patey, D. H., and Scarff, R. W. (1928). The position of histology in the prognosis of carcinoma of the breast. Lancet, 1, 801-804.

Patey, D. H., and Scarff, R. W. (1929). Further observations on the histology of carcinoma of the breast. Lancet, 2, 492-494.

Richardson, W. W. (1956). Medullary carcinoma of the breast. Brit. J. Cancer, 10, 415-423.

Stein, A. A. (1967). Carcinoma in situ of the breast: a review. In Pathology Annual, pp. 47-75. Butterworths, London.

Tribe, C. R. (1965). Cytological diagnosis of breast tumours by the imprint method. J. clin. Path., 18, 31-39.

Webb, A. J. (1970). The diagnostic cytology of breast carcinoma. Brit. J. Surg., 57, 259-264.

\section{Reports and Bulletins prepared by the Association of Clinical Biochemists}

The following reports and bulletins are published by the Association of Clinical Biochemists. They may be obtained from The Administrative Office, Association of Clinical Biochemists, 7 Warwick Court, Holborn, London, WC1R 5DP. The prices include postage, but airmail will be charged extra. Overseas readers should remit by British Postal or Money Order. If this is not possible the equivalent of 50p is the minimum amount that can be accepted.

\section{SCIENTIFIC REPORTS}

3 Automatic Dispensing Pipettes. An assessment of 35 commercial instruments 1967 P. M. G. BROUGHTON, A. H. GOWENLOCK, G. M. WIDDOWSON, and K. A. AHLQUIST $80 \mathrm{p}(\$ 2)$

4 An Evaluation of five Commercial Flame Photometers suitable for the Simultaneous Determination of Sodium and Potassium March 1970 P. M. G. BROUGHTON and J. B. DAWSON $80 \mathrm{p}(\$ 2)$

SCIENTIFIC REVIEWS

1 The Assessment of Thyroid Function March 1971 F. V. FLYNN and J. R. HOBBS 60p (\$1.50)

2 Renal Function Tests Suitable for Clinical Practice January 1972 F. L. MITCHELL, N. VeALL, and R. W. E. WATTS $60 \mathrm{p}(\$ 1.50)$

\section{TECHNICAL BULLETINS}

9 Determination of Urea by AutoAnalyzer November 1966 RUTH M. HASLAM 40p (\$1)

11 Determination of Serum Albumin by AutoAnalyzer using Bromocresol Green October 1967 B. E. NORTHAM and G. M. WIDDOWsON 40p (\$1)

13 An Assessment of the Technicon Type II Sampler Unit March 1968 B. C. GRAY and G. K. McGOWAN 40p (\$1)

14 Atomic Absorption Spectroscopy. An outline of its principles and a guide to the selection of instruments May 1968 J. B. DAWSON and P. M. G. BROUGHTON 40p (\$1)

15 A Guide to Automatic Pipettes (2nd edition) June 1968 P. M. G. BROUGHTON 40p (\$1)
16 A Guide to Automation in Clinical Chemistry May 1969 P. M. G. BROUGHTON 60p (\$1.50)

17 Flame Photometers (2nd edition) 1969 P. WILDING $60 \mathrm{p}(\$ 1.50)$

18 Control Solutions for Clinical Biochemistry (4th edition) March 1970 P. M. G. BROUGHTON 60p (\$1.50)

19 Spectrophotometers. A comparative list of low-priced instruments readily available in Britain May 1970 C. E. WILDE and P. SEWELL $60 \mathrm{p}(\$ 1.50)$

20 Quantities and Units in Clinical Biochemistry June 1970 P. M. G. BROUGHTON 60p (\$1.50) More than 30 copies in units of 10 at 20p

21 Filter Fluorimeters: A comparative list of 18 instruments September 1970 H. BRAUNSBERG and S. S. BROWN 60p (\$1.50)

22 Bilirubin standards and the Determination of Bilirubin by Manual and Technicon AutoAnalyzer Methods January 1971 BARBARA BILLING, RUTH HASLAM, and N. WALD $60 \mathrm{p}(\$ 1.50)$

23 Interchangeable Cells for Spectrophotometers and Fluorimeters September 1971 E. S. BROWN and A. H. GOWENLOCK 60p $(\$ 1.50)$

24 Simple Tests to Detect Poisons March 1972 B. w. MEADE et al. $60 \mathrm{p}(\$ 1.50)$

25 Blood Gas Analysers May 1972 k. DIXoN 60p (\$1.50)

26 Kits for Enzyme Activity Determination September 1972 S. B. ROSALKI and D. TARLOW 80p $(\$ 2.00)$

27 Assessment of Pumps Suitable for Incorporation into Existing Continuous Flow Analytical Systems November 1972 A. FLECK et al 60p (\$1.50) 